\title{
A Transmission Probability Control Algorithm Based on Competing Node Estimation in MANET
}

\author{
Shentao Wang*, Xiaoli Liu, Jingwen Sui, Shengze Qiang, Rui Li and Junqiang He \\ Chongqing Institute of Communication, Chongqing 400035, China \\ ${ }^{*}$ Corresponding author
}

\begin{abstract}
Most of Transmission Probability Control Algorithms in Mobile Ad hoc Network (MANET) research are based on the assumption that the number of competing nodes is known by every node. With an increasing number of wireless devices or appliances, the number of competing node in network is changed dynamically, so the assumption that the number of competing nodes is known by every node is no longer suitable. A transmission probability control algorithm, based on competing node estimation, is proposed in this paper. The simulation results show that the protocol can estimate the number of competing node, control the transmission probability dynamically and decrease the end-to-end delay.
\end{abstract}

Keywords-transmission probability control; competing node estimation ; manet

\section{INTRODUCTION}

Over the past few years, research in MANET has yielded considerable advances, notably in the areas of new routing and medium access techniques. Yet, significant shortcomings of ad hoc networks remain, especially compared with wire network.

MANET suffers from fundamental capacity limitations, connectivity/survivability problems, and is prone to jamming. Attempts to surmount these problems at the medium access, network by way of innovative and often ingenious protocols have yielded only incremental success. In this paper, we consider the transmission probability control enhancement---based on the estimation of competing nodes' number to address the above problems and provide a significant improvement in network end-to-end delay.

Currently, Transmission probability control algorithms are based on prior knowledge of the number of its neighbor nodes in the network, Such as literature [1-5], CSMA/CD (Carrier Sense Multiple Access/Collision Detect) based on the transmission probability control, CSMA is divided into persistent CSMA and non - persistent CSMA. Persistent CSMA Continuously monitors the channel to channel idle, and it is also divided into 1-persistent CSMA and p-persistent CSMA. 1-persistent CSMA will not send the frame immediately until the channel is free, p-persistent CSMA sends data with the probability $\mathrm{p}$, and after a period of delay with the probability of 1-p starts monitor channel, if the channel is idle, starts the sending process with probability $\mathrm{p}$, until the channel is busy or frame is send successfully. With the popularity of personal intelligent mobile terminal equipment, the number of competing node in network is becoming more and more difficult to know, research in transmission probability control based on prior knowledge of the number of its neighbor nodes in the network already can't meet the needs of the reality.

\section{CE-TPC Algorithm}

In this paper, MANET is a synchronous Time Division Multiple Access network. In a slot, node obtains channel with the probability $P_{G}$, channel being idle with probability $P_{I}$, channel conflicts with probability $P_{C}$, there are k competing nodes in the network, the transmission probability is p, so $P_{G}$, $P_{I}, P_{C}$ can be expressed as:

$$
\left\{\begin{array}{l}
P_{I}(k, p)=(1-p)^{k} \\
P_{G}(k, p)=k p \cdot(1-p)^{k-1} \\
P_{C}(k, p)=1-P_{I}(k, p)-P_{G}(k, p)=1-(1-p+k p)(1-p)^{k-1}
\end{array}\right.
$$

Based on Poisson theorem, formula 1can be approximate to:

$$
\left\{\begin{array}{l}
P_{I}(k, p)=e^{-k p} \\
P_{G}(k, p)=k p \cdot e^{-k p} \\
P_{C}(k, p)=1-(1+k p) e^{-k p}
\end{array}\right.
$$

The partial derivatives of formulation 2 as shown in formulation 3 ,

$$
\begin{aligned}
& \left\{\begin{array}{l}
\frac{\partial P_{I}(k, p)}{\partial p}=-k \cdot e^{-k p}<0 \\
\frac{\partial P_{I}(k, p)}{\partial k}=-p \cdot e^{-k p}<0
\end{array}\right. \\
& \left\{\begin{array}{l}
\frac{\partial P_{C}(k, p)}{\partial p}=k^{2} p e^{-k p}>0 \\
\frac{\partial P_{C}(k, p)}{\partial k}=k p^{2} e^{-k p}>0
\end{array}\right.
\end{aligned}
$$

From formulation 3, free slot probability $P_{I}$ is monotonically decreasing with transmission probability $p$ and the number of competing nodes $k$. From formulation 4, collision slot probability $P_{C}$ is monotonically increasing with transmission probability $p$ and the number of competing nodes 
$k$. By the opposite monotone property of transmission probability $p$ and the number of competing nodes $k$, it is known that collision slot and free slot are fluctuant within a range. So the collision slot and free slot length thresholds of are set as: $T h_{C}=P_{C} \cdot F 、 T h_{I}=P_{I} \cdot F, F$ is data slot length.

So, the number $k_{i+1}$ of competing nodes in next frame is calculated as shown in formulation 5.

$$
k_{i+1}=k_{i}-N_{G i}+N_{C i}-T h_{C}-N_{I i}+T h_{I}
$$

Where $N_{G i} 、 N_{C i} 、 N_{I i}$ are amount of obtained slot, amount of collision slot and amount of free slot respectively.

As there is one successful slot, 0 or multiple collision slots and free slots in every successful communication, the communication delay is as shown in formulation 6 :

$$
\begin{aligned}
\text { Delay } & =T_{1} \cdot \frac{P_{I}}{P_{G}}+T_{C} \cdot \frac{P_{C}}{P_{G}}+T_{G} \\
& =T_{1}\left[\frac{1-\frac{T_{C}}{T_{I}}+\frac{T_{C}}{T_{I}} \cdot e^{k p}}{n p}+\left(\frac{T_{G}}{T_{I}}-\frac{T_{C}}{T_{I}}\right)\right]
\end{aligned}
$$

Where $T_{C}$ is collision slot, $T_{I}$ is free slot, $T_{G}$ is slot of obtained channel.

The partial derivatives of formulation 6 as shown in formulation 7and 8,

$$
\begin{gathered}
\frac{\partial \text { Delay }}{\partial p}=T_{G} e^{k p}(k p-1)+T_{G}-T_{I} \\
\frac{\partial \partial \text { Delay }}{\partial \partial p}=k^{2} T_{G} p e^{k p}>0
\end{gathered}
$$

From formulation 8, it can be known that Delay has minimum value as the function of Delay is concave function, while the formulation 8 meets conditions as follows:

$$
\frac{\partial \text { Delay }}{\partial p}=T_{G} e^{k p}(k p-1)+T_{G}-T_{I}=0
$$

From formulation 9 it can be known that,

$$
e^{k p}(1-k p)=\frac{T_{C}-T_{I}}{T_{C}}
$$

In differential application area, when $|x|$ goes to zero, $e^{x} \approx 1+x$. So formulation 10 can be rewritten as follows:

$$
\left(1-k p_{1}\right)\left(1+k p_{1}\right)=\frac{T_{C}-T_{I}}{T_{C}}
$$

$$
p_{1}=\frac{\sqrt{T_{I} / T_{C}}}{k}
$$

From formulation 12, we can see that the transmission probability can be regulated dynamically by the amount of network competing nodes in CE-TPC algorithm.

\section{ANALYSIS OF CE-TPC ALGORITHM}

To validation the performance of CE-TPC algorithm, it is compared with p-CSMA/CD algorithm based on OPNET network simulation software.

\section{TABLE I. SIMULATION PARAMETERS OF CE-TPC ALGORITHM}

\begin{tabular}{|c|c|}
\hline Parameters & Values \\
\hline Number of competing node & $1 \sim 20$ \\
\hline Network range & $400 \mathrm{~m} \times 400 \mathrm{~m}$ \\
\hline $\begin{array}{c}\text { Initialization value of } \\
\text { transmission probability }\end{array}$ & 0.5 \\
\hline Simulation time & $10 \mathrm{~min}$ \\
\hline Speed of mobility & $0-10 \mathrm{~m} / \mathrm{s}$ \\
\hline Duration of collision slot & $200 \mu \mathrm{s}$ \\
\hline
\end{tabular}

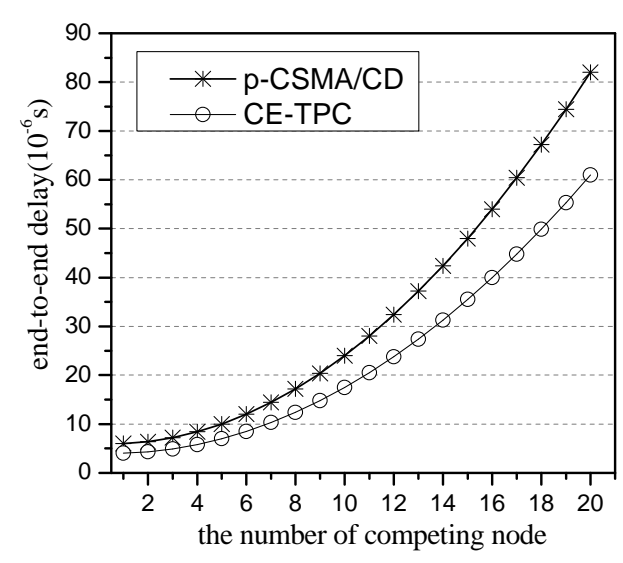

FIGURE I. END-TO-END delay 


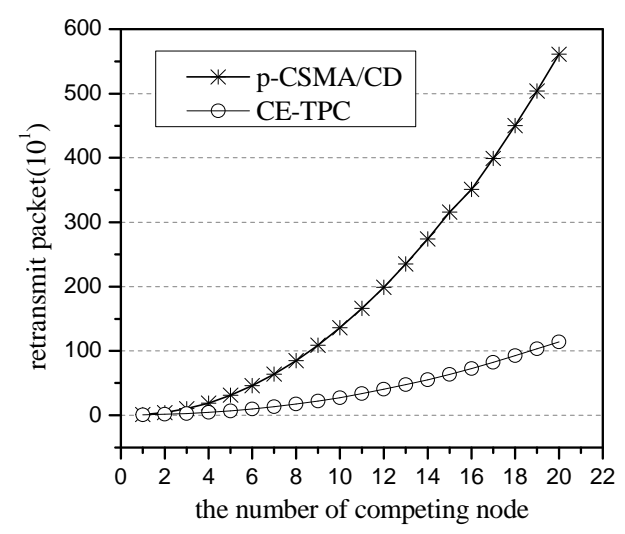

FIGURE II. RETRANSIMITTING PACKET

From Figure I and Figure II, it is can be seen that the number of retransmitting packet sharply increased in pCSMA/CD algorithm and slot utilization decreased when network competing nodes added; However, as the transmission probability can be regulated dynamically by the number of competing nodes in CE-TPC algorithm, the collision probability decreased, then the number of retransmitting packet and the increasing amplitude were both less than in pCSMA/CD algorithm, and the end-to-end delay in CE-TPC algorithm is also less than in p-CSMA/CD algorithm.

\section{CONCLUSION}

In this paper, a mobile Ad hoc network transmission probability control algorithm CE-TPC was proposed, the number of competing nodes can be calculated quickly, and the end-to-end delay can be decreased by the transmission probability regulated dynamically based on the calculated number of competing nodes.

\section{ACKNOWLEDGMENT}

This work is supported by Laboratory capacity improvement program (No: cstc2014pt-sy0010), Chongqing college and universities good science and research translation significant project (No: KJZH14112).

\section{REFERENCES}

[1] C.K.Siew, M.H.Er. An Adaptive Algorithm for performance optimization of modified p-persistent CSMA/CD local area network].IEEE, Control and Power Engineering Proceedings. TENCON '93[J]:175-177.

[2] HE Wei, NAN Jing-chang, PAN Feng. Improved Dynamic p-Persistent CSMA Protocol. Computer Engineering, 2010, 36(11).P.118-120.

[3] Fang Chen, Liu Hao, Shi Longxing. Congestion alleviation strategy based on adaptive contention window for wireless sensor networks. JOURNAL OF SOUTHEAST UNIVERSITY (Natural Science Edition), 2013, 43(4).P.686-690.

[4] BRUNO R, CONTI M, GREGORI E. Optimization of efficiency and energy consumption in p-persistent CSMA-based wireless LANs. IEEE Transactions on Mobile Computing, 2002, 1(1):10-31.

[5] BRUNO R, CONTI M, GREGORI E. Optimal capacity of p-persistent CSMA protocols. IEEE Communication Letters, 2003, 7(3): 139-141. 